\title{
Nutritional composition and antioxidant properties of Canarium odontophyllum Miq. (dabai) fruits.
}

\begin{abstract}
The present study sought to determine the nutritional composition and antioxidant properties of Canarium odontophyllum Miq. (dabai) fruits from different districts in Malaysia, namely Kanowit, Sarikei, Kapit and Song in Sarawak. Two varieties of the fruits were investigated. Lipid ( $21.16 \pm 4.71$ to $25.76 \pm 3.03 \mathrm{~g} / 100 \mathrm{~g} \mathrm{FW})$ was the major macronutrient in dabai fruits, while the predominant minerals were calcium, sodium and potassium. The fruit protein was rich in aspartic and glutamic acids which accounted for $45-49 \%$ of total amino acids. Purple dabai fruits from Kapit were found to contain the highest total phenolic levels, flavonoids and anthocyanin contents $(\mathrm{p}<0.01)$ and to exhibit the most significant antioxidant activities $(\mathrm{p}<$ 0.01 ), using trolox equivalent antioxidant capacity (TEAC) and ferric reducing ability (FRAP) assays. Antioxidant activities were highly correlated with total phenolic and flavonoid contents of dabai fruits.
\end{abstract}

Keyword: Canarium odontophyllum; Indigenous fruit; Nutritional composition; Antioxidant properties; Food analysis; Food composition. 